\title{
Textual analysis of the discourse of language for specific purposes on the example of French language of international relations
}

\section{[Analyse textuelle du discours de la langue de specialité sur l'exemple de la langue francaise des relations internationales]}

\author{
Larisa Spynu - Irina Shlepova - Olga Kanygina
}

DOI: $10.18355 /$ XL.2019.12.01XL.03

\begin{abstract}
This article is devoted to the problem of correlation between the foreign language for specific purposes (LSP) and the common language in the linguistic context. The purpose of the study is to analyze the discourse of the LSP at the linguistic level, to reveal its essential characteristics and to determine its role and place in the system of the common language. To achieve the goal of the research, the authors have chosen a textual analysis of the discourse of the French language of international relations as a methodological basis. As a material for the analysis, the authors selected authentic texts of various subjects, which are typical for the discipline.
\end{abstract}

Key words: textual analysis, language for specific purposes, discourse analysis, textual linguistics, international relations

Annotation : L'article est consacré au problème du rapport entre la langue étrangère de spécialité et la langue couramment utilisée dans un contexte linguistique.

Le but de cette étude est d'analyser le discours de la langue étrangère de spécialité au niveau linguistique et d'identifier ses caractéristiques essentielles afin de déterminer son rôle et sa place dans le système de la langue couramment utilisée.

Les auteurs ont choisi pour base méthodologique l'analyse textuelle du discours du français des relations internationales. Des textes authentiques de divers sujets typiques de cette spécialité ont été sélectionnés pour l'analyse.

Mots clés : analyse textuelle, langue de spécialité, analyse du discours, linguistique, textuelle, relations internationales

\section{Introduction}

Depuis longtemps, la langue française confirme son importance au niveau mondial et suscite l'intérêt de l'étudier dans de nombreux domaines. Selon l'Organisation internationale de la Francophonie, le français est classé deuxième langue des affaires en Europe et troisième dans le monde (official site of Francophonie). La nécessité d'utiliser une langue étrangère dans la communication professionnelle tende à l'apparition et au développement qualitatif de nouvelles approches pour l'apprentissage $\mathrm{du}$ français dans diverses professions. Ainsi, de nombreux établissements d'enseignement supérieur ne limitent pas leurs programmes de formation aux cours de langue étrangère générale, mais ils essaient d'inclure et de développer des programmes de formation de la langue étrangère à visée de la communication professionnelle.

\subsection{Particularités de l'apprentissage de la langue de spécialité}

L'étude de la discipline de la langue de spécialité pendant de nombreuses années est toujours le sujet pertinent et insuffisamment étudié. La confirmation de cette disposition est la publication d'un certain nombre d'ouvrages scientifiques consacrés à ce problème, aussi bien en science russe qu'à l'international (Lerat, 1997 ; Mangiante, 2006 ; Zmeyeva, 2014 ; Spynu, 2016). 
La langue de spécialité ne se limite pas à l'étude de la terminologie, mais représente l'utilisation des connaissances de base d'une langue étrangère afin d'exprimer et de transférer des connaissances professionnelles spéciales en langue étrangère.

Lors de l'étude de la langue de specialité (LS), l'un des principaux problèmes pour les spécialistes est la définition du terme de langue de spécialité en sciences. Dans la science linguistique russe, il est généralement accepté d'utiliser la définition de «la langue de spécialité » comme l'ensemble des moyens linguistiques utilisés dans un domaine de communication spécialisé et limité afin d'assurer la compréhension mutuelle des acteurs. (Bagrova, 1998). Selon le linguiste français P. Lerat, la langue de spécialité ne doit pas être opposée à la langue générale, mais c'est la langue générale qui est complétée par le lexique spécialisé et est employé à des fins professionnelles (Lerat, 1997).

La langue française possède une variété de termes pour désigner le processus d'apprentissage d'une langue étrangère à des fins professionnelles : le « français de spécialité », le « français sur objectifs spécifiques/FOS », le «français professionnel», le « français à visée professionnelle », le « français langue professionnelle ».

Les auteurs posent le problème de définir la notion de la langue de spécialité (le « français de spécialité ») et ses caractéristiques particulières. Contrairement au « cours général de français » du français langue étrangère (FLE), qui comprend un ensemble de règles et le lexique général usuel, conçu pour communiquer dans des situations quotidiennes (Barthélémy, 2007: 191), le français de spécialité est «la base de ressources et de méthodes pédagogiques pour de divers domaines d'activité professionnelle (spécialités) utilisée par les enseignants pour développer leurs propres cours de formation concernés » (Zmeyeva, 2014 : 43). La langue de spécialité suppose l'approche générale de la discipline ou de la sphère d'activité professionnelle, l'orientation au public le plus nombreux et cherche à prendre en compte la diversité professionnelle existante (Mangiante, Parpette, 2004).

Le linguiste français J.-M. Mangiante met en évidence les caractéristiques suivantes de la langue de spécialité :

- l'étude d'une seule spécialité dans une large gamme ;

- la formation à long terme ;

- une variété de sujets et de compétences liés à la discipline ;

- le contenu du cours est un nouveau matériel pour l'enseignant;,

- le travail de l'enseignant est basé sur les exigences de la spécialité étudiée et non sur les besoins des étudiants ;

- des connaissances générales de la langue de spécialité sont enseignées (mais parfois insuffisantes) ;

- l'évaluation interne du programme de formation (Mangiante, 2006).

Dans cet article, les auteurs n'ont pas le but d'étudier en détail le problème de la polyvalence de la langue française de spécialité, mais il est inacceptable de ne pas tenir compte de ses caractéristiques essentielles. Les particularités susmentionnées émettent des exigences qualitativement nouvelles pour le développement et la mise en œuvre du processus éducatif pour les étudiants et pour les enseignants.

\subsection{Le concept de discours}

Pour la théorie et la pratique de l'étude de la langue étrangère, il est important de comprendre le concept de discours. Il existe de nombreux points de vue sur le terme « discours ». Le discours est souvent considéré comme un travail langagier qui ne se limite pas à une déclaration concrète. Nous partageons le point de vue de la chercheuse $\mathrm{V}$. Rostovtseva, qui suggère que le discours est un phénomène de communication complexe, qui comprend, en plus du texte, des facteurs extralinguistiques (connaissance du lieu, du but d'un destinataire) (Rostovtseva, 2009 : 261). Cependant, à notre avis, les facteurs extralinguistiques ne se limitent pas à cet ensemble et incluent absolument les paramètres de la réalité sociale (non 
linguistique). Ainsi, chaque discours est caractérisé par un chronotope, c'est-à-dire l'unité des paramètres spatiaux et temporels. Le concept de chronotope a été justifié par M. Bakhtine (Barkhtine, 1986) et est utilisé productivement dans de nombreuses études du discours. Il pensait que l'essentiel dans la notion du chronotope est l'orientation axiologique de l'ensemble «espace-temps ", dont le rôle est d'exprimer la position personnelle, le sens. Selon M. Bakhtine, les sens contenus dans l'œuvre ne peuvent être objectivés que par leur expression spatio-temporelle. De plus, l'auteur, l'œuvre et le lecteur ont leurs propres chronotopes.

Selon la catégorisation de A.M. Laurian (Laurian, 1983), J. Pearson (Pearson, 1998), I. Meyer et K. Mackintosh (Meyer et Mackintosh, 1996), qui est basée sur la situation de communication, les types de discours se distinguent selon le "producteur » du discours, le « destinataire » du discours, le but du discours et le niveau de composante technique ou spéciale du discours.

Quelle que soit la définition, la plus grande différence réside dans le discours luimême, c.-à-d. dans sa spécialité : discours de relations internationales, de politique, de droit, de sciences naturelles, etc. Il est nécessaire de diviser le discours en différents systèmes conceptuels si nous reconnaissons l'idée que chaque discours reflète les caractéristiques conceptuelles de différents domaines de la connaissance. (Rey, 1979)

\subsection{Place des composantes extralinguistiques dans le discours diplomatique}

Ces dernières années, le monde a connu des changements importants liés à la mondialisation, à l'intégration, aux progrès scientifiques et technologiques rapides et aux bouleversements politiques et sociaux. En même temps, les frontières entre les sphères de l'interne et de l'externe de la vie publique deviennent de plus en plus floues, ce qui est reflété directement dans l'aggravation des discussions entre les Etats. Autrement dit, l'humanité actuelle existe dans une situation de changement continu et de déstabilisation. Ces facteurs se reflètent dans la langue et leurs traces se sont inévitablement manifestées au niveau de la communication sous forme écrite et orale. Ces changements ont ouvert aujourd'hui de vastes possibilités dans le domaine des relations internationales et ont permis de couvrir de nouveaux domaines de communication. Ces faits ont eu un impact significatif sur la communication et sur le discours diplomatique.

L'étude de ces phénomènes du point de vue de l'analyse linguistique, du point de vue de la communication stratégique, de l'étude des moyens linguistiques, d'une part, et de l'analyse extralinguistique, de l'autre, permet d'examiner d'autres aspects du fonctionnement de l'interaction internationale. Le discours diplomatique moderne a acquis un caractère interdisciplinaire.

La preuve en est une série d'exemples confirmant que les connaissances brillantes d'une langue étrangère, la connaissance de son vocabulaire, des règles de grammaire et de stylistique ne sont pas suffisantes pour une communication réussie (orale ou écrite, verbale ou non verbale), en particulier pour réussir les négociations internationales et obtenir les résultats souhaités.

Sinon, il pourrait y avoir des cas de fausse compréhension du sens des déclarations et, par conséquent, d'utilisation incorrecte de mots, par exemple :

"Sous les auspices"

La Syrie et Israël ont signé quatre séries de négociations de paix indirectes sous les auspices de la Turquie.

Dans cette phrase, " sous les auspices » est équivalent au mot "médiateur », c'est-àdire cette phrase pourrait être :

La Syrie et Israël ont signé quatre séries de négociations de paix indirectes dont la Turquie a été médiateur. (Spynu, Sheremetyeva, 2012 : 116).

L'incapacité à utiliser des techniques de LS, le manque de maîtrise de la PE, de la PO et de la communication, du comportement dans la sphère professionnelle

XLinguae, Volume 12 Issue 1XL, January 2019, ISSN 1337-8384, eISSN 2453-711X 
diplomatique et, par conséquent, la violation des règles du protocole international peut conduire à une rupture des négociations ou même à une rupture des relations entre les pays.

Des problèmes similaires se sont posés à maintes reprises lors de l'interaction de représentants de différentes cultures, groupes ethniques et nationalités. La nécessité de résoudre et de prévenir les conflits interculturels, ethniques et interreligieux a incité les gouvernements de nombreux pays d'organiser pour leurs diplomates (avant de les envoyer à l'étranger pour travailler) des cours obligatoires de culturologie, d'étiquette et d'histoire d'un futur pays d'accueil. De nombreuses universités offrant une formation dans le cadre du programme «Relations internationales » ont adopté la pratique de stages linguistiques annuels pour les futurs fonctionnaires de départements internationaux dans les pays où ils reviendront ultérieurement comme diplomates.

Par exemple, l'Université RUDN a une bonne expérience de nombreuses années dans la coopération avec un certain nombre d'établissements d'enseignement supérieur de premier plan dans le monde : stages linguistiques, programmes de formation inclusive, doubles masters (RUDN: rudn.ru).

Cette expérience vise à donner une idée de la «connaissance de fond», ce qui implique une compréhension des réalités de la vie d'un pays ainsi que de la vision du monde inhérente à sa population.

La connaissance et le respect des règles de l'étiquette du pays sont nécessaires à établir des contacts diplomatiques, simplifient considérablement le processus de communication interethnique et interétatique.

\subsection{Caractéristiques du discours diplomatique}

"Un diplomate c'est quelqu'un qui réfléchit deux fois avant de ne rien dire" - F. Sawyer, 1822-1891, (dicocitations.lemonde.fr).

Avant d'analyser les particularités du discours diplomatique, il faut se souvenir de la définition de l'institution de la diplomatie donnée par le linguiste suisse P. Serio, spécialiste en analyse du discours. Il dit que l'institution de la diplomatie, c'est tout organisme qui impose des restrictions à l'effet de la fonction d'énonciation; ce sont le statut du sujet de l'énoncé et du destinataire, des types de contenu de ce qui peut et doit être dit, ainsi que des circonstances de la déclaration d'un énoncé qui sont licites pour tel ou tel poste (Serio, $1999: 29)$.

Serio élargit la compréhension du terme «diplomatie » en introduisant le terme discursif «statut» (statut du sujet de l'énoncé et statut du destinataire) comme élément nécessaire de la situation extralinguistique. En outre, il est important de souligner que l'activité diplomatique institutionnelle « impose des limitations à l'effet de la fonction d'énonciation ». Il semble que ces restrictions concernent des genres et des situations discursives (circonstances de la déclaration d'énonciation), ainsi que des stratégies spécifiques.

En outre, la caractéristique la plus importante du discours diplomatique est la présence des relations spatio-temporelles susmentionnées (cadre spatio-temporel), c.-à-d. chronotope.

Chaque discours se déroule dans un espace et dans un temps déterminés (Wodak, 1997 : 8). Cette déclaration peut être entièrement attribuée au discours diplomatique. Le chronotope est considéré dans le cadre de la catégorie de contenu informationnel communicatif et pragmatique, qui est causée par la situation communicative du discours diplomatique. Le cadre institutionnel strict impose à l'expéditeur de fournir au destinataire des informations fiables, pertinentes et non ambiguës. Puisque le sujet de nos recherches est le français de spécialité dans le domaine des relations internationales où l'un des principaux moyens de communication est le texte écrit, c.à-d. moyens de communication écrits indirects, les informations sur la relation spatiotemporelle sont extrêmement importantes. La précision ainsi que la clarté de la 
compréhension sont obtenues grâce à l'utilisation de dates et de toponymes spécifiques, qui découle des exemples ci-dessous.

Dans le discours diplomatique en tant qu'entité institutionnelle on peut distinguer les stratégies et techniques discursives suivantes: une stratégie de politesse visant à coopérer et à prévenir les conflits éventuels, une stratégie argumentative comme partie de la catégorie communicative et pragmatique de la persuasion.

"Le style diplomatique ", écrit $\mathrm{H}$. Wildner, "doit être distingué avant tout par la simplicité et la clarté ; on n'entend pas la simplicité du mode d'expression artisanale, mais la forme classique de la simplicité, qui permet de choisir pour chaque objet le seul mot approprié dans les circonstances » (Wildner, 1959 : 9).

Ces stratégies sont mises en œuvre à l'aide de certains moyens linguistiques dans certaines tactiques. Par exemple, la tactique de distanciation, la tactique de dosage de l'information et de sa pertinence, la tactique d'explication de la cause, la tactique de la gratitude, la tactique de la bienveillance, etc.

Dans les ouvrages consacrés à l'étude du discours diplomatique, on a bien sûr porté son attention sur les stratégies et la tactique du discours diplomatique. Ainsi, dans l'œuvre de E.A. Weber (Weber, 2004 : 137), on explore des stratégies visant à réduire la dissonance cognitive : une stratégie de la création de l'ambiguïté, la création d'une incertitude intentionnelle, une stratégie de l'utilisation des moyens de rectitude politique. À son tour, T.A. Volkova (Volkova, 2007 : 169) examine les stratégies de discrédit, de présentation de soi et de manque de sincérité, conformément au thème général de sa recherche de thèse, le caractère stratégique de la traduction dans le discours diplomatique. L'ouvrage du scientifique français S. Villar «Discours diplomatique » (Villar, 2006) se situe à la jonction de la sémiotique, de l'analyse du discours et de la sociologie, où les tactiques examinées, notamment celle de l'ambiguïté et de l'insincérité passent à travers le prisme de deux niveaux de sémantique et de pragmatique.

Chacun des pays participant à la communication internationale a sa propre structure sociale, son histoire, ses traditions, sa culture et sa langue. La communication entre les Etats serait impossible sans respecter les principes fondamentaux communs des relations mutuelles, tels que le respect de la souveraineté, l'égalité, l'intégrité territoriale, la non-ingérence dans les affaires intérieures des uns et des autres. Le protocole diplomatique est l'outil par lequel les Etats appliquent ces principes dans la pratique. Les auteurs du livre «Cérémonie diplomatique et protocole », J. Wood et J. Serre, indiquent que « en général, un protocole est un ensemble de règles de conduite pour les gouvernements et leurs représentants à des occasions officielles et non officielles » (Wood, Serre, $1974: 18$ ). Le Dictionnaire diplomatique donne une définition plus large de ce terme : «Un protocole diplomatique est une combinaison de règles généralement acceptées, de traditions et de conventions observées par le gouvernement, des départements des affaires étrangères, représentants diplomatiques, responsables dans la communication internationale » (DD: 443).

Ainsi, le protocole diplomatique revêt un caractère normatif et universel : les participants de l'activité diplomatique devraient suivre les standards, quelles que soient la structure de l'État et la situation de la politique étrangère. En liaison avec le rôle du protocole de communication internationale, les linguistes ont examiné le phénomène de conventionalité dans le discours diplomatique. À son tour, S. Villar introduit le terme «code d'institution » et suggère la présence de langage interne, faisant référence aux spécificités de la communication dans le cadre du discours diplomatique, soumises aux normes du protocole (Villar, 2006 : 34). Par exemple, «l'administration centrale du MAE », en France, est appelée le « Département » par ceux qui y travaillent.

Outre le protocole, il existe un certain nombre d'autres documents régissant les activités des services diplomatiques : règlements du droit international, 
principalement les «Conventions de Vienne sur les relations diplomatiques (1961) et consulaires » (1963), dispositions et articles de la Constitution, décrets pertinents du décret du chef de l'État et du gouvernement pays, arrêtés et directives du ministre des Affaires étrangères, autres actes réglementaires et juridiques définissant les activités de l'appareil des organes de l'État en ce qui concerne leur exécution par un diplomate international des fonctions fondées sur les principes généralement reconnus du droit international et des traités internationaux du pays.

En outre, le discours diplomatique comprend les types de textes suivants : note, note verbale, mémorandum, lettre diplomatique, lettres de créance, lettres de rappel, etc.

Certains des types de documents susmentionnés existant dans le domaine des relations internationales ont été pris comme base de nos recherches afin de comprendre la nature du discours diplomatique.

\section{Etude expérimentale. Analyse des textes}

Nous avons choisi pour base méthodologique de notre étude une analyse textuelle du discours du français dans le domaine des relations internationales.

L'avantage de cette méthode réside dans la possibilité d'étudier la langue française de spécialité à plusieurs niveaux : lexico-sémantique, grammatical, morphologique, syntaxique, ainsi que la capacité de prendre en compte et d'analyser les éléments extralinguistiques mentionnés ci-dessous, qui sont invariablement présents dans le discours et en sont inséparables.

Pour l'analyse textuelle, nous avons sélectionné une douzaine de textes authentiques (voir Annexe) rédigés en français dans le domaine des relations internationales (site officiel de l'ONU). Les textes couvrent divers sujets liés aux relations internationales, tels que : le droit international, les organisations internationales, la lutte contre le terrorisme, l'égalité des sexes, le climat et l'écologie.

\subsection{Niveau lexico-sémantique}

En termes lexico-sémantiques, ces textes contiennent à la fois un vocabulaire commun pour les relations internationales et celui plus spécifique visé à caractériser un sujet particulier concerné (en fonction de la thématique des textes).

\begin{tabular}{|l|l|}
\hline Lexique général & $\begin{array}{l}\text { Organisations internationales } \\
\text { traité } \\
\text { communauté internationale }\end{array}$ \\
\hline Lexique thématique & $\begin{array}{l}\text { non-prolifération } \\
\text { droits de l'homme } \\
\text { prise d'otages } \\
\text { réchauffement climatique }\end{array}$ \\
\hline Métaphores & $\begin{array}{l}\text { etre au cœur de } \\
\text { être couronné de }\end{array}$ \\
\hline
\end{tabular}

\subsection{Niveau morphologique}

$\mathrm{Au}$ niveau morphologique, on voit souvent des noms verbaux des verbes, ainsi que la formation des mots par de préfixes et de suffixes (adverbes de manière).

Cela confère au discours un caractère strict et officiel.

\begin{tabular}{|l|l|}
\hline Noms verbaux & $\begin{array}{l}\text { promotion } \\
\text { déclaration } \\
\text { prise }\end{array}$ \\
\hline Formation des mots par ajout de préfixes & $\begin{array}{l}\text { dé-nucléarisation } \\
\text { ré-affirmer } \\
\text { dé-ploiement }\end{array}$ \\
\hline Formation des mots par ajout de suffixes & largement \\
\hline
\end{tabular}




\begin{tabular}{|l|l|}
\hline & également \\
& fortement \\
\hline Acronymes & ONU \\
& UE \\
& CE \\
& AG \\
& OCDE \\
& OTAN \\
\hline
\end{tabular}

Il faut noter que les verbes et les constructions qui véhiculent une action, ainsi que les structures adverbiales, sont communs à tous les textes analysés.

\subsection{Niveau de grammaire}

Lors de l'analyse des textes de la langue de spécialité des relations internationales, il a été révélé que ces textes sont caractérisés par les caractéristiques grammaticales suivantes :

- l'utilisation du Présent, du Passé Composé et du Futur simple.

- les constructions impersonnelles et passives sont fréquemment utilisées ;

- l'utilisation de gérondif

- l'utilisation du subjonctif

\section{Niveau grammatical}

\begin{tabular}{|l|l|}
\hline Présent & $\begin{array}{l}\text { 1. L'activité des Nations Unies couvre toutes les } \\
\text { parties du globe. } \\
\text { 2. Le Soudan continue de requérir l'attention de la } \\
\text { communauté internationale. } \\
\text { 3. La Charte des Nations Unies préconise } \\
\text { expressément le développement progressif du droit } \\
\text { international et sa codification. }\end{array}$ \\
\hline Passé Composé & $\begin{array}{l}\text { 1. En 2010, l'Organisation des Nations Unies a } \\
\text { accueilli la première Conférence d'examen des } \\
\text { Parties au Traité sur la non-prolifération des armes } \\
\text { nucléaires (TNP). } \\
\text { 2. Les événements qui ont traversé le Moyen- } \\
\text { Orient et l'Afrique du Nord cette année ont } \\
\text { démontré une fois de plus que le désir de droits de } \\
\text { 1'homme ignore les frontières géographiques et } \\
\text { culturelles. } \\
\text { 3. Le Conseil a invité les États Membres à } \\
\text { accélérer l'échange de renseignements sur les } \\
\text { mouvements terroristes. }\end{array}$ \\
\hline Futur simple & $\begin{array}{l}\text { 1. Le Groupe proposera des solutions qui viseront } \\
\text { à faire sortir la population de la pauvreté et à } \\
\text { renforcer l'équité et l'égalité des chances tout en } \\
\text { préservant la planète qui nous porte. } \\
\text { 2. Cette initiative permettra d'arrêter les grandes } \\
\text { orientations. }\end{array}$ \\
\hline $\begin{array}{l}\text { Constructions } \\
\text { impersonnelles }\end{array}$ & $\begin{array}{l}\text { 1. Il est indispensable d'assurer l'accès universel à } \\
\text { 1'énergie durable. } \\
\text { 2. Il convient désormais d'intensifier les efforts. } \\
\text { 3. Il faut notamment mettre en ouvre la Stratégie }\end{array}$ \\
\hline
\end{tabular}

XLinguae, Volume 12 Issue 1XL, January 2019, ISSN 1337-8384, eISSN 2453-711X 


\begin{tabular}{|l|l|}
\hline & $\begin{array}{l}\text { mondiale pour la santé des femmes et des } \\
\text { enfants... }\end{array}$ \\
\hline Forme passive & $\begin{array}{l}\text { 1. L'Organisation des nations unies a été fondée... } \\
\text { 2. Son rapport final, qui sera remis en janvier... } \\
\text { 3. ...des armes nucléaires (TNP) qui ait été } \\
\text { couronnée de succès }\end{array}$ \\
\hline Gérondif & $\begin{array}{l}\text { 1. En renforçant nos moyens de diplomatie } \\
\text { préventive et en soutenant les processus axés sur } \\
\text { une paix durable } \\
\text { 2. ...en effectuant des enquêtes et en punissant } \\
\text { chaque acte de violence, en protégeant les } \\
\text { femmes... }\end{array}$ \\
\hline Subjonctif & $\begin{array}{l}\text { 1. ...afin que l'Organisation puisse rapidement } \\
\text { élever le niveau de ses opérations sur le terrain... } \\
\text { 2. Il faut que l'Accord de paix global de 2005 qui a } \\
\text { mis fin à la guerre civile soit respecté et que toutes } \\
\text { les questions en suspens soient résolues. } \\
\text { 3. Il est absolument essentiel que les transitions } \\
\text { démocratiques en Tunisie et en Égypte réussissent. }\end{array}$ \\
\hline
\end{tabular}

\subsection{Niveau de syntaxe}

Lorsque l'on étudie la syntaxe des textes de la langue de spécialité des relations internationales, il convient de souligner l'utilisation de phrases déclaratives; les phrases interrogatives et les exclamations sont rarement utilisées dans de tels textes. Dans les textes de la langue de spécialité des relations internationales prévalent des phrases subordonnées complexes. Dans les textes prévalent des phrases avec un ordre de mots direct : Sujet - Prédicat - membres secondaires de la phrase, cependant, il existe des phrases avec inversion :

\begin{tabular}{|c|c|}
\hline $\begin{array}{l}\text { Phrases à plusieurs } \\
\text { subordonnées }\end{array}$ & $\begin{array}{l}\text { 1. M. Emmerson a expliqué qu'une partie } \\
\text { importante de son travail de Rapporteur spécial } \\
\text { consistait à revoir les législations nationales et } \\
\text { les définitions du terrorisme qui sont courantes } \\
\text { et utilisées dans le domaine juridique pour } \\
\text { s'assurer que les Etats n'abusent pas de la lutte } \\
\text { contre le terrorisme à des fins politiques. } \\
\text { 2. Si les opérations de maintien et de } \\
\text { consolidation de la paix, de prévention des } \\
\text { conflits et d'assistance humanitaire sont bien } \\
\text { connues, l'influence des Nations Unies et de son } \\
\text { système (institutions spécialisées, fonds et } \\
\text { programmes) se manifeste également de } \\
\text { multiples façons dans notre quotidien et } \\
\text { contribue àcréer un monde meilleur. }\end{array}$ \\
\hline Ordre de mots direct & $\begin{array}{l}\text { L'organisation des nations unies a été fondée en } \\
\text { 1945, après la seconde guerre mondiale, par } 51 \\
\text { pays déterminés à maintenir la paix et la sécurité } \\
\text { internationales, à développer des relations } \\
\text { amicales entre les nations, à promouvoir le } \\
\text { progrès social, à instaurer de meilleures } \\
\text { conditions de vie et à accroître le respect des } \\
\text { droits de l'homme. }\end{array}$ \\
\hline
\end{tabular}




\section{Résultats}

Les résultats de l'étude confirment notre hypothèse selon laquelle le discours diplomatique suppose non seulement la présence et l'utilisation de vocabulaire spécial mais aussi son utilisation dans des situations communicatives particulières communes de ce discours. Ce qui nous permet de conclure sur l'importance de l'approche d'apprentissage/enseignement de la langue de spécialité, en tenant compte du chronotope et d'autres paramètres non linguistiques, tels que la connaissance de l'orientation, les objectifs du destinataire.

À la suite de l'analyse des textes, il s'est avéré que les caractéristiques qualitatives du discours diplomatique sont les suivantes : vocabulaire spécialisé, utilisation de phrases déclaratives complexes au Présent, Passé Composé et Futur simple, utilisation de constructions impersonnelles et passives, le subjonctif et le conditionnel.

Tout cela permet de rendre la langue des relations internationales plus précise, plus concrète et d'éviter l'ambiguïté, ce qui est très important pour la langue de la communication diplomatique.

Pour l'enseignant, le français de spécialité, conçu et enseigné sur la base d'une approche actionnelle et communicative et en matière d'analyse discursive, présente un intérêt considérable tant au niveau pédagogique que méthodologique. Serait-il donc possible de passer du concept de langage de la spécialité au concept de discours spéciaux.

\section{Remerciement}

Le travail a été effectué avec le soutien financier du ministère de l'éducation et de la science de la Fédération de Russie pour le Programme d'amélioration de la compétitivité du RUDN "5-100" parmi les principaux centres scientifiques et éducatifs du monde 2016-2020.

\section{Bibliographic references}

BAGROVAYA A. 1998. Formation of Communicative Skills In Reading. Communicative Orientation of Teaching Foreign Languages in a Non-Language University. Moscow: MGLU. 44-50 pp.

BAKHTINE M. 1986. Forms of time and chronotope in the novel. Essays on historical poetics. Moscow. 234-407 pp.

BARTHÉLÉMY F. 2007. Professeur De FLE : Historique, Enjeux Et Perspectives / Fabrice Barthélémy. Paris : Hachette. - P. 191.

CONVENTIONS DE VIENNE SUR LES RELATIONS DIPLOMATIQUES (1961), Available online:

http://legal.un.org/ilc/texts/instruments/french/conventions/9_1_1961.pdf.

CONVENTIONS DE VIENNE SUR LES RELATIONS DIPLOMATIQUES (1963), Available online:

http://legal.un.org/ilc/texts/instruments/french/conventions/9_2_1963.pdf

DIPLOMATIC DICTIONARY Vyshinskij A. YA., Lozovskij $\overline{\mathrm{S}}$. A. - M.:

Gosudarstvennoe izdatel'stvo politicheskoj literatury, 1948., Available online:

http://niv.ru/doc/dictionary/diplomatic/index.htm.

LA FRANCOPHONIE, Available online: https://www.francophonie.org/3e-languedes-affaires-dans-le.html.

LAURIAN A.-M. 1983. Typologie Des Discours Scientifiques : Deux Approches. Dans Etudes De Linguistique Appliquee, No 52. Paris: Didier Erudition, P. 8-20.

LE DICTIONNAIRE DES CITATIONS, LE MONDE (Edition mondiale). Available online: https://dicocitations.lemonde.fr .

LERAT P. 1997. Approches Linguistiques Des Langues Spécialisées, Asp, 15-18 p.

XLinguae, Volume 12 Issue 1XL, January 2019, ISSN 1337-8384, eISSN 2453-711X 
MANGIANTE J.-M. 2006. Francais De Specialite Ou Francais Sur Objectif Specifique: Deux Demarches Didactiques Distinctes.

MANGIANTE, J-M. PARPETTE, C. 2004. Le Francais Sur Objectif Specifique: De L'analyse Des Besoins a L'elaboration D'un Cours . Paris: Hachette, Collection F.

MEYER I. ET MACKINTOSH K. 1996. The Corpus From A Terminographer's Viewpoint. International Journal Of Corpus Linguistics, P. 257-285.

ORGANISATION INTERNATIONALE DES NATIONS UNIES, Available online: http://www.un.org/fr/index.html.

PEARSON J. 1998. Terms In Context. Amsterdam: John Benjamins Publishing Company.

PEOPLES' FRIENDSHIP UNIVERSITY OF RUSSIA (RUDN), Available online: http://eng.rudn.ru/.

REY A. 1979. La Terminologie, Noms Et Notions. Paris: Presses Universitaires De France. Que Sais-Je ?, N ${ }^{\circ} 1780$. P. 44.

ROSTOVCEVA V. 2009. Competence and Competences: The Hermeneutic Aspect In the Context of the Diversification of Modern Education. Tomsk. Tpu:Kit.

SERIO P. 1999. Comment on lit des textes en France. Quadrature de sens. L'Ecole francaise de l'analyse du discours. Moscow : Progress. P. 29.

SPYNU L. 2016. Features of Teaching Foreign Language Specialties. SantPetersbourg : Globalniy Naychniy potancial № 12 (69) P. 47-49.

SPYNU L., SHEREMETYEVA O., 2012. Lire la presse en français, Moscow: Universitetskaya kniga. P. 116.

VILLAR C. 2006. Le discours diplomatique. Paris: L'Harmattan, coll. Pouvoirs compares. $284 \mathrm{p}$.

VOLKOVA T. 2007. Diplomatic discourse in terms of strategic translation and communication: on the material of the English and Russian languages. Chelyabinsk.

P. 169.

WEBER E. A. 2004. Experience of linguistic study of cognitive dissonance in English diplomatic discourse. Irkutsk. P.137.

WILDNER H. 1959. Die Technik der Diplomatie: L'art de négocier. Vienna: Springer-Verlag. P. 9.

WODAK R. 1997. Language. Discourse. Politics. Volgograd : Peremena. P. 8.

WOOD J., SERRES J. 1976. Diplomatic ceremonial and protocol. Moscow: Progress. P. 18.

ZMEYEVA T. 2014. Concept of Professionally-Oriented Foreign Language Teaching in French Linguodidactic. Materials of the Eighth International Scientific and Practical Conference. Nizhny Novgorod Institute of Management - Branch of the Federal State Budgetary Educational Institution of Higher Professional Education "Russian Academy of National Economy and Public Administration under the President of the Russian Federation”. 42-46 pp.

Words: 8226

Characters: 54624 (30,35 standard pages)

Assoc. prof. Spynu Larisa

Department of Foreign Languages

Faculty of Humanities and Social Sciences

Peoples' Friendship University of Russia

6 Miklukho-Maklaya Street,

117198, Moscow

Russia

spynu-lm@rudn.ru

Assistant, Shlepova Irina 
Department of Foreign Languages

Faculty of Humanities and Social Sciences

Peoples' Friendship University of Russia

6 Miklukho-Maklaya Street,

117198, Moscow

Russia

Shlepova-is@rudn.ru

Assistant, Kanygina Olga

Department of Foreign Languages

Faculty of Humanities and Social Sciences

Peoples' Friendship University of Russia

6 Miklukho-Maklaya Street,

117198, Moscow

Russia

Kanygina-om@rudn.ru 


\section{Annexe}

\section{L'ORGANISATION INTERNATIONALE DES NATIONS UNIES}

L'Organisation des nations unies a été fondée en 1945, après la seconde guerre mondiale, par 51 pays déterminés à maintenir la paix et la sécurité internationales, à développer des relations amicales entre les nations, à promouvoir le progrès social, à instaurer de meilleures conditions de vie et à accroître le respect des droits de l'homme.

De par son statut unique à l'échelon international et les pouvoirs que lui confère sa Charte fondatrice, l'Organisation peut prendre des mesures pour résoudre un grand nombre de problèmes. En outre, elle constitue un forum où ses 193 États Membres expriment leur opinion par l'intermédiaire de l'Assemblée générale, du Conseil de sécurité, du Conseil économique et social, des autres organes et comités.

L'activité des Nations Unies couvre toutes les parties du globe. Si les opérations de maintien et de consolidation de la paix, de prévention des conflits et d'assistance humanitaire sont bien connues, l'influence des Nations Unies et de son système (institutions spécialisées, fonds et programmes) se manifeste également de multiples façons dans notre quotidien et contribue à créer un monde meilleur.

L'Organisation se consacre à un grand nombre de questions fondamentales, comme le développement durable, la protection de l'environnement et des réfugiés, les secours en cas de catastrophe, la lutte contre le terrorisme, le désarmement et la nonprolifération, la promotion de la démocratie, les droits de l'homme, l'égalité des sexes et la promotion de la femme, la gouvernance, le développement économique et social, la santé publique, le déminage et l'augmentation de la production alimentaire et bien plus encore.

Ce faisant, elle s'attache à atteindre les objectifs fixés et à coordonner les efforts afin de créer un monde plus sûr pour les générations présentes et futures.

\section{DÉVELOPPEMENT DURABLE ET SANS EXCLUSIVE}

Les enjeux d'aujourd'hui sont multiples : alimentaires, énergétiques, financiers, hydriques, climatiques. Nous sommes face au défi des trois 50 : en 2050, la planète comptera plus de 9 milliards d'habitants, soit $50 \%$ de plus par rapport au début du millénaire, et le volume mondial des émissions de gaz à effet de serre devra avoir diminué de $50 \%$ par rapport à l'an 2000. Il est temps, aujourd'hui plus que jamais, de s'interroger sur les facteurs qui favorisent l'instabilité et de trouver des moyens plus durables d'assurer la croissance et la prospérité pour tous au XXIe siècle.

Le développement durable n'est pas un choix mais une nécessité. C'est aussi un moyen historique de faire advenir une économie plus équitable, résistante et rejetant peu de carbone, une économie véritablement adaptée aux réalités du XXIe siècle. C'est sur ces questions que doit réfléchir le Groupe de haut niveau sur l'écoviabilité mondiale que j'ai créé en 2010 et qui rassemble une dizaine d'experts des secteurs public et privé et de la société civile. Le Groupe proposera des solutions qui viseront à faire sortir la population de la pauvreté et à renforcer l'équité et l'égalité des chances tout en préservant la planète qui nous porte. Son rapport final, qui sera remis en janvier 2012, viendra alimenter les travaux de la Conférence Rio +20 et ceux d'autres mécanismes intergouvernementaux.

Il est indispensable d'assurer l'accès universel à l'énergie durable si l'on veut relever deux des défis les plus pressants de notre époque : réduire la pauvreté et écarter autant que faire se peut les dangers potentiels que les changements climatiques font peser sur notre sécurité. C'est à cette fin que je lance aujourd'hui l'Initiative en faveur d'une énergie durable pour tous, dont la vocation est de mobiliser $\mathrm{c}$ et les entreprises privées en vue d'élargir l'accès à l'énergie, d'améliorer le rendement énergétique et d'accroître les sources d'énergie renouvelable. Cette initiative permettra d'arrêter les grandes orientations et les décisions d'investissement nécessaires pour engager les pays dans une voie énergétique plus durable au cours des 20 prochaines années. 
En 2012, à Rio de Janeiro, tout juste 20 ans après l'historique Sommet planète Terre, les dirigeants du monde auront l'occasion rare de placer le développement durable au cœur de l'élaboration des politiques internationales. La Conférence Rio +20 sera le moment idéal de promouvoir la durabilité et de construire aujourd'hui l'économie de demain, une économie plus équitable et reposant sur les énergies propres.

Le sommet de l'année dernière sur les objectifs du Millénaire pour le développement a marqué une avancée décisive, avec l'établissement d'un plan d'action quinquennal pour atteindre les objectifs du Millénaire, sensibiliser la population mondiale et susciter les engagements nécessaires en matière de politiques et de moyens pour remédier aux carences et insuffisances.

Il convient désormais d'intensifier les efforts. Il faut notamment mettre en œuvre la Stratégie mondiale pour la santé des femmes et des enfants et poursuivre la lutte contre le VIH/sida, le paludisme, la tuberculose et autres maladies non transmissibles, en particulier en Afrique.

Il faut également renforcer l'assistance apportée aux pays les moins avancés, dont les besoins en matière d'énergie propre, de sécurité alimentaire et nutritionnelle et de travail décent sont les plus grands.

\section{UN MONDE PLUS SUR}

Je continue de m'employer à renforcer la capacité de l'Organisation à mener à bien la prévention des conflits, l'instauration de la paix, le maintien de la paix et la consolidation de la paix. En renforçant nos moyens de diplomatie préventive et en soutenant les processus axés sur une paix durable, nous construirons des solutions à long terme et réagirons plus efficacement aux conflits.

\section{SOUDAN}

Le Soudan continue de requérir l'attention de la communauté internationale. Il faut que l'Accord de paix global de 2005 qui a mis fin à la guerre civile soit respecté et que toutes les questions en suspens soient résolues.

En juillet 2011, le pays a vécu un moment historique lorsque le Sud est officiellement devenu indépendant, franchissant ainsi l'une des dernières étapes du processus enclenché par l'Accord de paix global. Ce résultat faisait suite à un référendum qui s'est déroulé sans incident majeur. L'Organisation est prête à faire en sorte que ce tout nouveau pays puisse être viable.

Des questions difficiles subsistent, concernant notamment le tracé des frontières, la libre circulation des personnes et le partage des ressources essentielles. Le statut de la zone contestée d'Abyei n'a pas été résolu et les tensions débordent sur le reste du Kordofan méridional. Nous devons redoubler d'efforts pour parvenir à une solution qui concilie les intérêts de tous.

$\mathrm{Au}$ Darfour, l'opération conjointe Union africaine-ONU de maintien de la paix s'emploie tout à la fois à réduire la violence et les souffrance sur le terrain et à trouver une solution diplomatique qui soit garante d'une paix durable.

\section{AFRIQUE DU NORD ET MOYEN-ORIENT}

Les événements historiques de 2011 en Afrique du Nord et au Moyen-Orient posent, y compris à l'Organisation des Nations Unies, la difficile question de savoir comment réagir efficacement à cette possibilité, qui ne s'offre qu'une fois par génération, de soutenir les aspirations populaires à un changement démocratique dans le monde arabe.

L'Organisation continuera de condamner la violence et d'encourager les dirigeants dans toute la région à respecter les droits de l'homme et à répondre aux aspirations légitimes de leur peuple à un changement par un dialogue sans exclusive et de véritables réformes, et non par la répression.

Il est absolument essentiel que les transitions démocratiques en Tunisie et en Égypte réussissent. Il s'agit là de processus qui relèvent du national, mais l'Organisation 
continuera d'offrir son aide et ses compétences en matière de transition et d'élections démocratiques, ainsi que de développement économique équitable et sans exclusive.

En Lybie, nous avons mené une action décisive de protection des civils et nous sommes disposés à apporter notre aide pour les grands problèmes de la phase postconflit. Nous appuierons les efforts du peuple libyen dans sa marche vers un avenir pacifique et démocratique sous le signe de l'unité nationale, de la réconciliation sociale et de la reprise économique.

Dans l'intervalle, le processus de paix au Moyen-Orient ne saurait rester immobile alors qu'une vague de changements balaie toute la région. La profonde méfiance entre Palestiniens et Israéliens est toujours là. L'Organisation continuera d'exhorter les deux parties à reprendre sans plus tarder les négociations, dans l'intérêt des deux peuples, et à négocier sérieusement de manière à parvenir à une paix juste, durable et globale, c'est-à-dire à deux États vivant côte à côte dans la paix et la sécurité.

\section{DROITS DE L'HOMME ET RESPONSABILISATION}

La reconnaissance du fait qu'il existe des droits de l'homme universels est l'un des trois piliers de l'Organisation des Nations Unies. La possibilité pour chaque être humain d'exercer, sur un pied d'égalité, ses droits inaliénables constitue le fondement de la liberté, de la justice et de la paix dans le monde et revêt une importance capitale pour la sécurité et le développement à l'échelle mondiale.

Je me suis souvent exprimé sur les droits de l'homme et les libertés fondamentales et je me suis engagé à agir avec les États Membres pour sauvegarder ces droits et promouvoir le droit international des droits de l'homme. Le Haut-Commissariat des Nations Unies aux droits de l'homme s'emploie à faire en sorte que ces droits soient intégrés aux politiques des États Membres et de l'Organisation. Il se consacre à veiller à ce que les violations de ces droits soient portées à l'attention de la communauté internationale et que leurs auteurs aient à rendre compte de leurs actes.

Les événements qui ont traversé le Moyen-Orient et l'Afrique du Nord cette année ont démontré une fois de plus que le désir de droits de l'homme ignore les frontières géographiques et culturelles. Nous avons tous le désir d'un monde dans lequel tout homme, femme ou enfant vit dans la dignité, à l'abri du besoin, de la peur et de la faim, un monde sans violence ni discrimination, garantissant l'accès au logement, aux soins de santé, à l'éducation et à un avenir.

L'Organisation des Nations Unies est en train de mener le monde vers une ère de la responsabilisation. Les élections et leurs suites en Côte d'Ivoire et les procès et les sentences du Tribunal pénal international pour le Rwanda et celui pour l'ex Yougoslavie montrent que les puissants ne peuvent plus escompter l'impunité en cas de violation grave des droits de l'homme.

La décision de la communauté internationale de protéger les civils confrontés à la violence de leur propre gouvernement constitue une étape décisive dans l'application de la doctrine encore en évolution dite de la « responsabilité de protéger».

\section{DÉSARMEMENT ET NON-PROLIFÉRATION NUCLÉAIRES}

En 2010, l'Organisation des Nations Unies a accueilli la première Conférence d'examen des Parties au Traité sur la non-prolifération des armes nucléaires (TNP) qui ait été couronnée de succès depuis 10 ans. Les États-Unis d'Amérique et la Fédération de Russie ont également conclu un nouveau Traité START prévoyant la réduction de leurs arsenaux nucléaires déployés.

Il est d'une importance capitale que le Traité sur l'interdiction complète des essais d'armes nucléaires entre en vigueur et que le TNP soit pleinement appliqué. Les États devraient également relancer les travaux de la Conférence du désarmement.

Je demeure profondément attaché à la dénucléarisation de la péninsule coréenne et je partage le désir de la communauté internationale de régler la situation concernant l'Iran. Je réaffirme également la nécessité d'une zone exempte d'armes nucléaires au Moyen-Orient. 
Au lendemain de l'accident nucléaire de Fukushima, j'exhorte les gouvernements à collaborer avec les organisations internationales pour prendre des mesures concrètes propres à renforcer la sûreté nucléaire.

\section{RÉAGIR AUX GRANDES CRISES HUMANITAIRES}

En 2010, le monde a connu des catastrophes humanitaires massives - depuis le tremblement de terre en Haïti jusqu'aux inondations qui ont ravagé une bonne partie du Pakistan. L'ampleur de ces crises a donné une idée de la nécessité pour l'Organisation et ses partenaires humanitaires de continuer de tout faire pour améliorer la coordination des secours.

J'ai constamment appelé à faire davantage pour veiller à ce que les ressources soient utilisées de la manière la plus efficace possible, afin que l'Organisation puisse rapidement élever le niveau de ses opérations sur le terrain et pourvoir aux besoins des populations.

J'ai pris la tête des appels à un financement accru des causes humanitaires, et j'ai insisté sur la nécessité d'un engagement aussi bien immédiat qu'à plus long terme sur le terrain. Au Soudan comme dans la corne de l'Afrique, j'ai pris la tête des appels à un accès humanitaire illimité aux zones touchées par les récents combats. Tant que les travailleurs humanitaires ne pourront pas distribuer des fournitures dont la population a désespérément besoin, il y aura lieu de craindre que la famine qui sévit actuellement dans certaines parties de la Somalie s'étende à d'autres zones.

J'ai aussi souligné la nécessité de ne jamais perdre de vue les crises humanitaires prolongées qui n'ont pas capté l'attention du reste du monde, notamment dans l'est de la République démocratique du Congo.

Cela étant, en dernière analyse, si l'on s'en tient à la réaction, on ne parviendra jamais à faire face aux besoins humanitaires émergents. C'est la raison pour laquelle je suis en train de remanier la stratégie de réaction humanitaire de l'Organisation pour l'axer sur le renforcement de nos moyens de venir en aide aux pays pour réduire ou atténuer les effets des crises par des mesures de prévention des conflits et de réduction des risques de catastrophe.

\section{L'ANTI-TERRORISME NE DOIT PAS EXCUSER LES VIOLATIONS DES DROITS DE L'HOMME}

La lutte contre le terrorisme ne doit pas servir de prétexte pour violer les droits de l'homme ou pour contraindre une opposition au silence tel que cela a été le cas dans plusieurs pays se voulant démocratiques au cours de la dernière décennie, a déclaré vendredi un expert de l'ONU.

"La plupart des Etats qui ont dû faire face aux problèmes de la lutte contre le terrorisme sous une forme ou une autre ont fait usage de méthodes qui sont incompatibles avec les droits de l'homme, » a dit le nouveau Rapporteur spécial des Nations Unies sur la lutte contre le terrorisme et les droits de l'homme, Ben Emmerson, devant la Troisième commission de l'Assemblée générale.

« Si je devais identifier le plus important principe à retenir, je dirai que c'est celui que le terrorisme en aucun cas ne doit être utilisé suivant une définition trop large qui permet aux Etat d'abuser du concept de sécurité nationale pour réduire l'opposition au silence, » a-t-il ajouté.

M. Emmerson a expliqué qu'une partie importante de son travail de Rapporteur spécial consistait à revoir les législations nationales et les définitions du terrorisme qui sont courantes et utilisées dans le domaine juridique pour s'assurer que les Etats n'abusent pas de la lutte contre le terrorisme à des fins politiques.

« Depuis dix ans, nous avons vu de nombreux Etats, y compris des Etats avec des traditions démocratiques et légales dont ils se disent fiers, prendre des mesures qui sont des violations systématiques de droits humains fondamentaux et reconnus au niveau international, » a indiqué $\mathrm{M}$. Emmerson.

XLinguae, Volume 12 Issue 1XL, January 2019, ISSN 1337-8384, eISSN 2453-711X 
« Nous avons également vu que les terroristes et ceux qui les soutiennent utilisent justement ces violations des droits de l'homme comme justification de leurs actes, » at-il ajouté.

Selon l'expert, la communauté internationale a compris que c'est en défendant les droits de l'homme que les Etats contribuent le plus efficacement à prévenir le terrorisme.

« La lutte contre le terrorisme qui respecte et promeut les droits de l'homme n'est pas seulement une question de principes, c'est également une question d'efficacité dans la prévention du terrorisme, » a expliqué le Rapporteur spécial.

Avant sa nouvelle nomination en tant que Rapporteur spécial, M. Emmerson a servi au Tribunal pénal international pour l'ex-Yougoslavie (TPIY), à la Cour pénale internationale (CPI) et à la Cour européenne des droits de l'homme.

« La philosophie qui anime mon mandat est qu'une action contre le terrorisme qui viole les droits de l'homme, ne sert pas à prévenir le terrorisme et depuis dix ans de telles pratiques ont exacerbé les griefs qui poussent les gens à faire les mauvais choix et à s'engager dans l'action terroriste, » a dit M. Emmerson.

\section{DROIT INTERNATIONAL}

La Charte des Nations Unies préconise expressément le développement progressif du droit international et sa codification. Les conventions, traités et normes - plus de 510 - résultant de ce travail sont autant d'instruments qui favorisent la paix et la sécurité internationales et le développement économique et social. Ils revêtent un caractère obligatoire pour les États qui les ratifient.

La Commission du droit international établit sur des sujets de droit international des projets de texte qui peuvent ensuite être adoptés sous forme de conventions ouvertes à la ratification des États. Certaines de ces conventions régissent les relations entre États: c'est le cas par exemple de la Convention sur les relations diplomatiques ou de la Convention sur l'utilisation des cours d'eau internationaux.

La Commission des Nations Unies pour le droit commercial international élabore des règles et des directives visant à harmoniser les lois qui régissent le commerce international et à faciliter leur adoption. L'ONU a également fait œuvre de pionnier en matière de droit de l'environnement. Le Programme des Nations Unies pour l'environnement administre des accords tels que la Convention sur la lutte contre la désertification, la Convention pour la protection de la couche d'ozone et la Convention sur le contrôle des mouvements transfrontières de déchets dangereux.

Le Protocole de Kyoto à la Convention-cadre des Nations Unies sur les changements climatiques, qui porte sur le problème du réchauffement planétaire, est entré en vigueur le 16 février 2005. Il fait obligation aux pays industrialisés de réduire leurs émissions combinées des six principaux gaz à effet de serre durant la période 20082012 en deçà des niveaux de 1990. Au moment de son entrée en vigueur, il avait été ratifié par 140 pays et à ce jour, par plus de 180 pays.

La Convention sur le droit de la mer vise à assurer à tous les pays l'accès aux richesses des océans dans des conditions équitables, à préserver ceux-ci de la pollution et à faciliter la liberté de navigation et la recherche. La Convention contre le trafic illicite de stupéfiants et de substances psychotropes est l'instrument international le plus important en matière de lutte contre le trafic des stupéfiants.

L'ONU est au cœur de toutes les activités internationales visant à créer un cadre juridique contre le terrorisme. Plus d'une douzaine de conventions internationales se rapportant à cette question ont été négociées sous ses auspices, notamment la Convention de 1979 contre la prise d'otages, la Convention de 1997 pour la répression des attentats terroristes à l'explosif et la Convention de 1999 pour la répression du financement du terrorisme; un traité global contre le terrorisme est en cours d'élaboration. 
En 2001, à la suite des attentats du 11 septembre contre les États-Unis, le Conseil de sécurité a adopté une résolution d'une large portée visant les terroristes, dans le cadre des dispositions cœrcitives de la Charte des Nations Unies. Cette résolution stipule notamment que tous les États doivent prévenir le financement des actes de terrorisme, ériger en infraction la fourniture de fonds destinés à ces actes et geler instantanément les avoirs financiers de ceux qui s'y livrent. Le Conseil a invité les États Membres à accélérer l'échange de renseignements sur les mouvements terroristes et décidé qu'ils devraient se prêter mutuellement la plus grande assistance lors des enquêtes criminelles et autres procédures portant sur les actes de terrorisme.

En savoir plus sur l'action de l'ONU contre le terrorisme.

\section{ONU : LA PREVENTION DE LA VIOLENCE CONTRE LES FEMMES EST UNE RESPONSABILITE COLLECTIVE}

10 octobre 2011 -La Rapporteuse spéciale des Nations Unies sur la violence contre les femmes, Rashida Manjoo, a qualifié lundi cette violence d'« envahissante, répandue et inacceptable où qu'elle se produise au monde ", lors de la présentation à l'Assemblée générale de l'ONU d'un nouveau rapport sur la violence commise contre les femmes, ses causes et ses conséquences.

"Que cela arrive en temps de paix ou de conflit, les différentes formes et manifestations de violence contre les femmes sont à la fois les causes et les conséquences de discriminations, d'inégalité et d'oppression. Mon rapport donne un aperçu des différentes formes de violence contre les femmes et comment les Etats devraient y répondre, » a déclaré Mme Manjoo.

La Rapporteuse spéciale a réitéré son appel au Etats pour qu'ils respectent leurs obligations internationales en matière de droits de l'homme concernant la prévention des actes de violence contre les femmes, en effectuant des enquêtes et en punissant chaque acte de violence, en protégeant les femmes contre la violence et en offrant un soutien aux victimes.

« Le combat pour les droits des femmes reste une obligation collective pour laquelle nous devons agir pour garantir que toutes les femmes du monde bénéficient pleinement de leurs droits, » a souligné Mme Manjoo. 